\title{
Y después vino "la dolarización"
}

Cuando en el año 2010 un ecuánime analista recoja los hechos principales de la presente y pasada década, podría titular así el capítulo tercero de su obra: "Y después vino la "dolarización"”. Sin duda, este historiador nos va a decir cosas que ahora ignoramos, porque estamos en la era de las promesas, de las dudas y de las posibles expectativas. Todo esto ha sido tan rápido, tan brusco, tan inconsulto, que nuestro historiador va a saber mucho más que nosotros. Pero nosotros podemos ayudarle a hacer arqueología histórica de los capítulos primero y segundo de la misma historia, porque nos ha tocado vivirlos. La economía tiene un orden y no hay capítulo tercero sin capítulos primero y segundo. Como gusta decir a los economistas, el capítulo tercero será una "coyuntura", un resultado futuro o conjunción de los hechos, políticas y medidas adoptadas por los gestores de los dos primeros capítulos, en este caso escritos por el mismo partido y los mismos gobiernos. Son capítulos interrelacionados e interdependientes.

Nosotros podemos decirle a nuestro historiador que inicie su capítulo tercero contando lo que ahora sentimos: que los resultados de este "golpe de timón" van a depender de múltiples conductas de grupos diferentes en nuestro país; los banqueros, los exportadores, los empresarios con mora bancaria, las señoras del mercado, la microempresa, los déficit fiscal y comercial, y también las estadísticas que siga emitiendo en Banco Central de Reserva para sostener el optimismo nacional. Creemos que mucho va a depender de las medidas que siga tomando Alan Greespan, atento vigilante de la "burbuja" de Estados Unidos y de los préstamos externos esperados, porque ahora navegamos con bandera de dólar. Como en el año 2010 será difícil encontrar diarios del año 2000, queremos recordarle a nuestro historiador cómo se presenta la ley de la integración monetaria en el discurso presidencial de Francisco Flores.

\section{El discurso presidencial}

Este discurso se inicia con algunas frases sorpresivas o enigmáticas: "Todos en el país sabemos que nuestra economía, después de lograr éxitos muy marcados, está experimentando un entrampamiento que ha generado un negativismo muy nocivo para el desarrollo del país". Como la palabra entrampamiento es muy fuerte, el presidente quiere deslindar culpabilidạdes. "No puede culparse a nuestros sectores productivos de este entrampamiento, ya que todas nuestras fuerzas económicas - los obreros, los empresarios, los hermanos en el extranjero, los agricultores- han dejado muy clara su-dedicación al trabajo y al desarrollo del país". La palabra entrampamiento la usa el presidente y la ha repetido en varias ocasiones el secretario técnico de la presidencia: "No podemos seguir esperando porque la economía ha llegado a un entrampamiento que, de no cambiarse, se volverá nocivo y puede, en algún momento, llegar a límites de recesión" (El Diario de Hoy, 24 de noviembre de 2000, p. 56).

Analicemos la palabra entrampamiento. Creo que todos admitimos que la economía está entrampada, es decir, que cayó en una trampa y no logra escapar de la trampa. La pregunta obvia es quién inventó y quién puso la trampa. Si el presidente Flores afirma que "no puede culparse a todas nuestras fuerzas económicas de este entrampamiento", 
sólo queda un gran culpable: el modelo económico y la conducción del modelo, timoneado por los tres últimos gobiemos. Esta es la conclusión lógica que se deriva del discurso presidencial, que deja un espacio de culpabilidad al sistema bancario, no citado entre las fuerzas económicas productivas. Algo de esto se observó en la tendencia de las más recientes elecciones. Se habló de voto de castigo.

\section{El capítulo primero}

Haciendo una breve historia, en la introducción del "Plan de Desarrollo Económico y Social, 1989-1994", se nos decía: "Una sociedad que quiere ser libre en el plan económico, político y en el orden de los valores y costumbres, debe apoyarse en los siguientes principios". Se citan cinco principios filosóficos y cuatro parámetros económicos, que aquí nos interesan más: la propiedad privada es condición necesaria para la eficiencia de la producción, el mercado libre asegura la mejor asignación de recursos, la competencia garantiza el funcionamiento del mercado y el Estado tiene un papel subsidiario. Estos eran los tres ejes del modelo que nos llevaría a una sociedad libre en lo económico, lo político y en el orden de los valores. El Estado sería sólo un "acólito" del modelo econónmico. Así comenzó el capítulo primero de aquella década conflictiva, donde los acuerdos de paz sí fueron un fuerte golpe de timón y lograron avances bien positivos. La palabra clave, en 1992, era “depuración" de ciertas instituciones públicas.

Sin embargo, la economía de mercado y la competencia cometieron un primer error de programación modélica. Se comenzó a reconstruir la casa por el tejado. El auge de 1992-1995 fue un auge del consumo, del comercio, asentado en importaciones y alentado por el crédito bancario con elevadas tasas de interés, que elevaron el tipo de cambio promedio a 8.99 colones. El Banco Central de Reserva tuvo que anclarlo a la tasa actual. Varias instituciones dieron su voz de alarma sobre este ineficiente, aunque jugoso, estilo de crédito. La Asociación Nacional de la Empresa Privada lo advertía en su Correo económico de octubre de 1994. "De darse un aumento en los mismos (encajes legales e intereses) los efectos sobre la inversión serían aún mayores que en la actualidad. Debe recordarse que, debido a la evoluciór. de la economía salvadoreña, los márgenes de ganancia en actividades dedicadas a la atención del comer- cio y de los servicios son mayores que en el resto, lo que de por sí es un sesgo en lo que se refiere a la dirección del crédito, en contra de las actividades industriales y de producción en general, ya que su mayor período de maduración y menor margen de ganancia las vuelve menos atractivas para el sistema financiero" ( $E C A, 1997$, p. 813).

Por las mismas fechas, la Fundación Salvadoreña para el Desarrollo Económico y Social (FUSADES), al comentar la política cambiaria, vuelve a subrayar el sesgo crediticio. "A manera de antecedentes cabe señalar que el panorama económico a finales de 1994 es positivo en términos de estabilidad de precios y de incremento real, con tasas cercanas al 5 por ciento. No obstante, el crecimiento productivo estuvo liderado por sectores de bienes no transables, a saber servicios, comercio y construcción. Dicho patrón de crecimiento no es sostenible a mediano y largo plazo, porque se da con menos participación de las principales actividades que generan valor agregado, divisas y empleo. Estas actividades corresponden a la actividad manufacturera y la agricultura, que son dos bases de la estructura productiva de exportación, o sea, de bienes transables, que es lo que podría mantener el desarrollo sostenido de la economía, según FUSADES lo ha venido señalando" (ibídem, p. 813). Estas son dos de las voces de alerta, cercanas al propio gobierno. En el presente año, Rafael Lemus lo ha dicho en forma lacónica: "el desempeño de la economía salvadoreña de los noventa tiene un fuerte nexo con la historia crediticia. El eje de consumo se instaló como factor preponderante del auge y agotamiento de la economía. Este consumo contó con un socio vulnerable, en tanto se apoyó en forma importante en un sobreendeudamiento bancario. La volatilidad de la economía es una réplica del comportamiento del consumo y crédito bancario" ("La industria bancaria de los noventa", FUSADES, agosto de 2000, p. 51).

En 1993, el Banco Central de Reserva publica las matrices insumo-producto de 1990. Estas matrices nos dan el entramado de todos los sectores productivos, como ramas interrelacionadas por sus mutuas ofertas y demandas de insumos, sus relaciones externas de importaciones y exportaciones, su respectiva gestación del valor agregado nacional... En Europa occidental se habían utilizado a modo de radar orientador de una planificación indicativa y concertada entre el Estado y los sectores productivos. Al hacer un análisis comparativo de 
las matrices de 1978 y 1990 se apreciaba una creciente terciarización económica, tal como lo indicaban ANEP, FUSADES, FUNDE, CENITEC y el IIES-UCA. Creíamos que el crédito bancario debía canalizarse hacia otros sectores productivos más dinamizantes de la economía, que relacionasen el multiplicador del crédito bancario con el multiplicador de la inversión, con miras al desarrollo de mediano y largo plazo ("Aportes económicos de las matrices $1978-1990$ a los programas de desarrollo 1994-1999", Realidad, 39). El sector bancario hizo oídos sordos a todos estos reclamos y las matrices insumo-producto dormían en las bodegas del Banco Central de Reserva. El auge ficticio del comercio y los servicios llevaba en su seno el germen del crecimiento frenado, que terminaría en el entrampamiento actual.

El recordado investigador Salvador O. Bran lo advertía al decir que el sistema financiero estaba orientado a sus propios fines. "Al aprovechar la desregulación, el sistema financiero se ha convertido en el sector más pujante del país, aunque no sea el más eficiente, lo cual puede comprobarse en los estados financieros que semanalmente publican, por ley, las mismas instituciones. Claro que eso no es criticable, al contrario, es la lógica del mercado; pero en las actuales circunstancias de desaceleración económica, el sistema debería efectuar una revisión de sus fortalezas, oportunidades, debilidades y amenazas, y reflexionar hasta cuándo podrá llegar la 'luna de miel' del predominio de las operaciones especulativas sobre las operaciones de apoyo a la producción nacional" ( $E C A$, 1997, p. 542). A tiempo se anunciaba el paso de la luna de miel a la mora bancaria. El mercado y la competencia no aseguraron la mejor asignación de recursos durante el primer capítulo de nuestra historia.

\section{Capítulo segundo}

En este escenario se inicia la historia del segundo gobierno de ARENA. Algo peculiar debe tener el mes de noviembre porque, al igual que en el presente año 2000 , en noviembre de 1994 , el ejecutivo económico, liderado por Manuel E. Hinds, se hizo presente en Washington para iinformar o consultar? el "golpe de timón" de la dolarización, de la reducción de aranceles, del alza del impuesto al valor agregado, de la privatización de servicios públicos. La publicidad oficial hablaba entonces del "pulgarcito centroamericano". El discurso presidencial del año 2000 anuncia que "tomaremos el liderazgo de Centroamérica...".

Una de las primeras acciones del gobierno Calderón Sol, léase Manuel E. Hinds, fue derruir y dispersar el Ministerio de Planificación y DesarroIlo Social, porque el Estado tiene sólo un papel subsidiario. El primer semestre de 1995 estuvo plagado de propuestas sorpresivas, algunas rechazadas por el sector empresarial (reducción de aranceles), otras por los sectores laborales (aumento del impuesto al valor agregado y privatización) y la dolarización criticada por bastantes académicos, incluido un antiguo presidente del Banco Central de Reserva. Como escribía Salvador O. Bran, sufrimos la inconsistencia de la política económica: "esto es indiscutible, pues al inicio del segundo gobierno de ARENA se dijo que El Salvador se convertiría en un gran país maquilador; a los pocos meses nadie habló sobre eso y se comenzó a promover la dolarización de la economía, proyecto que fue abandonado sin conocer las explicaciones; luego se dispuso liquidar la planificación global y enfatizar las políticas sectoriales. Esta acción también fue neutralizada y el propio Ministro de Hacienda, en forma insólita, declaró que las políticas sectoriales estaban 'pasadas de moda' y que no era productivo promoverlas. Así se llegó a la novedad de impulsar los clusters o sea las alianzas competitivas, que la mayoría de productores desconoce..." (ECA, 1997, pp. 538-539).

Cuando la economía ya descendía por el tobogán del crecimiento frenado, el gobierno presenta, en 1996, un "plan de gobierno 1994-1999", con el logolipo: "El Salvador un país de oportunidades". La publicidad oficial también utilizó otro subtítulo: "El Salvador país de propietarios". Pero la historia iba por otro lado; entre las argollas financieras y la concentración de la riqueza y del capital, se agregó un simple artículo al subtítulo: "El Salvador, un país de los propietarios" ("El bloque empresarial hegemónico salvadoreño", Tesis de grado, Universidad Centroamericana "José Simeón Cañas", noviembre de 2000). En esas estábamos cuando el presidente Calderón Sol, quien había liquidado la planificación global, anunció en sus discursos del 1 de mayo y del 1 de junio de 1997 que era preciso encomendar a un grupo de personalidades distinguidas la preparación de un plan nacional de desarrollo "con una visión de mediano y largo plazo, que nos lleve al desarrollo y genere estabilidad y confianza. Necesitamos tener 
una visión de largo plazo para dar estabilidad y continuidad al país, más allá de los períodos de gobierno, a fin de convertir a nuestro pais en un lugar atractivo para la inversión y generación de empleo, que eleven al bienestar y calidad de vida de la población". Esta era una paladina aceptación de que la propiedad, el mercado y la competencia, ejes del modelo, no habían engendrado ni estabilidad, ni confianza, ni continuidad en el mediano y largo plazo. Era necesaria la mano visible de un plan de nación.

Lo irónico es que no fue el Estado quien diseñó el plan de nación. Fueron otros grupos e instituciones los que editaron una secuencia de informes y programas económicos: Bases para un plan de nación, Temas claves para un plan de nación, Crecimiento estéril o desarrollo, Crecimiento con participación, Estado de la nación en desarrollo humano... Lo irónico es que el tercer gobierno de ARENA no les prestó ninguna atención hasta que en noviembre del 2000 "se apropia de las propuestas del plan de nación o que se subsuman inadecuadamente éstas al programa de integración". Con toda razón, Roberto Rubio, uno de los gestores de este plan territorial de nación, denuncia la usurpación gubernamental cuando dice estar en un proceso de concertación y al mismo tiempo de ocultamiento y destape sorpresivo de la integración monetaria (La Prensa Gráfica, 4 de diciembre de 2000 , p. 28). Tampoco este gobierno juega limpio. Por añadidura, niega que Manuel E. Hinds haya tenido arte y parte en el "golpe de timón", cuando sobran los testigos oculares que afirman lo contrario. Por supuesto, Manuel E. Hinds tiene pleno derecho a defender su teoría económica, abierta a discusión, como lo han venido haciendo otros profesionales, en repetidos desayunos de trabajo. Sin embargo, el gobierno incauta la "teoría" y la impone sin discusión.

Roberto Rivera Campos sintetiza el cierre de este segundo capítulo de ARENA en el final del siglo: "Consideraciones finales... la tendencia de la productividad media de los factores productivos a declinar, a la par de la disminución de la disponibilidad de capital por trabajador. La pérdida de productividad tendencial estaría en la base del bajo crecimiento logrado en el largo plazo. La persistencia de la pobreza es la otra gran característica de largo plazo de la economía... No cabe duda que en el corto plazo la política económica tiene un problema de inconsistencia, pues un régimen de tipo de cambio fijo requiere, entre otras cosas, mantener unas cuentas fiscales en equilibrio, o superavitarias si es posible, porque la política fiscal es el único instrumento de política económica disponible para hacer frente a los impactos negativos y amortiguar las fases recesivas del ciclo económico... Además, las cuentas fiscales deficitarias en sí mismas tienden a prolongar la recesión y a intensificarla por la presión al alza que ejercen sobre la tasa de interés..." ("La economía salvadoreña al final del siglo: desafíos para el futuro", FLACSO, febrero de 2000, pp. 344-245).

Esta es la economía "entrampada" que deja en herencia el segundo gobierno de ARENA al tercero. Si la economía está entrampada y necesita un fuerte golpe dé timón, cómo se puede decir a renglón seguido que "los esfuerzos de todos los salvadoreños han sido muy exitosos, al punto que ahora somos reconocidos internacionalmente como un país de libertades, de progreso social y de manejo económico responsable...". Amartya Sen no estaría muy de acuerdo con este concepto de "libertad". Por añadidura, el presidente Flores irrespeta las normas de la "propiedad intelectual" al decir en su discurso: "ayer, frente a los representantes de sectores productivos, partidos políticos, alcaldes, grupos gestores departamentales y

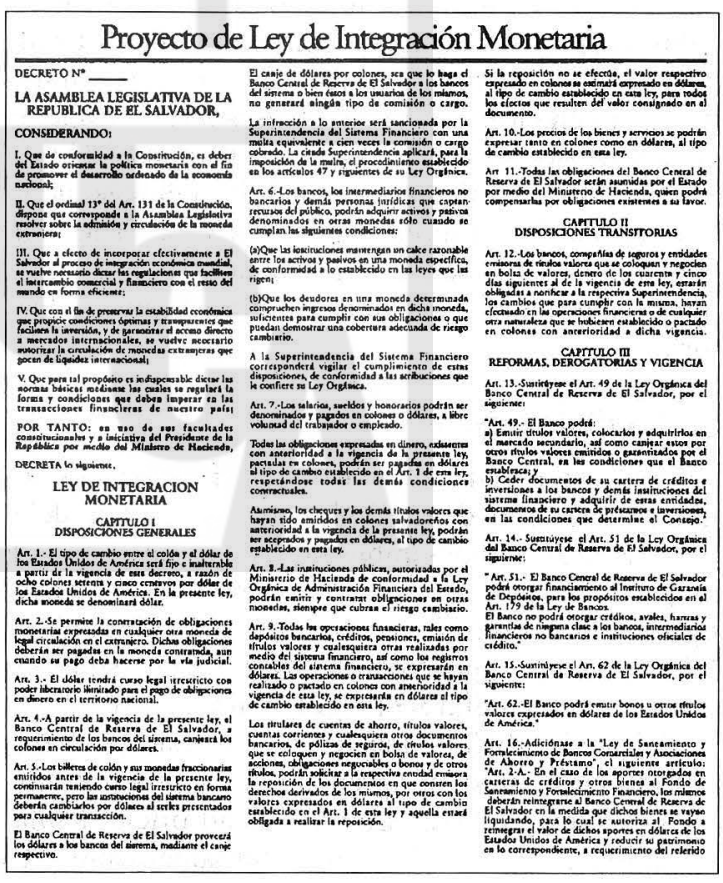


nuestro equipo de gobierno, anuncié mi compromiso de hacer realidad la integración territorial". Como ha denunciado Roberto Rubio, el plan territorial no es un "invento del equipo de gobiemo" y sus creadores se merecían al menos una mención de agradecimiento. Tal vez la Asociación Nacional de la Empresa Privada haya quedado un tanto consternada porque también su programa ENADE 2000 quedó en el "valle del olvido".

Aunque uno quisiera leer con ojos limpios este discurso, hay frases que enturbian la vista. "Nuestra política económica ha apuntado siempre a integrar a nuestro país al mundo en una economía sin privilegios, buscando el crecimiento de las exportaciones a través del aumento de la productividad". $\mathrm{He}$ aquí tres afirmaciones dudosas. Ni nuestra economía, ni la economía mundial globalizada se caracterizan por ser unas "economías sin privilegios". Por lo que atañe a nuestras exportaciones, ellas se ven gravadas por el mismo tipo de cambio fijado desde antes por el gobierno ( 1 dólar $=8.75$ colones.) y por la baja de la productividad o "disminución de la disponibilidad de capital por trabajador", demostrado en la investigación de Roberto Rivera Campos. Este modelo, al servicio de los servicios, ha reducido la productividad de los factores de producción. Estas afirmaciones restan sinceridad y verdad al discurso presidencial.

Se presenta la integración monetaria como la medida que "permitirá reducir las tasas de interés a niveles muy próximos a los internacionales". Pero esta afirmación silencia otra pregunta: ¿por qué subieron tanto las tasas de interés reales? Ello se debe, en buena parte, a dos políticas gubernamentales. La fijación y el mantenimiento del tipo de cambio (1 dólar por 8.75 colones), mediante el juego de compra y venta de dólares por parte del Banco Central de Reserva. El mismo banco en su desmedido afán por controlar todo amago de inflación, esponjó a ultranza la liquidez del sistema con miles de millones de CEM y CAM, con atractivas tasas de interés, forzando así el alza de los intereses reales. Actualmente, hay unos $7000 \mathrm{mi}-$ llones de títulos del Banco Central de Reserva y unos 4000 millones de Letes del Estado. La acuciosidad por mantener la política monetaria (el corto plazo) frenó el juego de la política financiera. Este es uno de los temas analizado econométricamente por Roberto Rivera Campos en su acápite: "Mal holandés y tasa de interés real" (ibídem, pp. 122-141).
Hasta aquí le hemos dado algunos apuntes de arqueología histórica a nuestro analista del año 2010, aunque tal vez encuentre otras lecturas numinosas de nuestro pasado reciente. Lo cierto es que la profecía del modelo de propiedad privada más mercado libre más libre competencia más Estado "subsidiario del mercado" nos ha llevado a una economía "entrampada", tal como lo afirma el presidente Flores. Tenemos serios presentimientos de que el "golpe de timón" tampoco sea la solución, porque ya nos han defraudado varias veces con los mismos "apocalipsis": el cielo nuevo y la tierra nueva... Desconocemos el futuro y sólo podemos decir algo del presente inmediato.

\section{4. ¿Integración o desintegración monetaria?}

Nunca entendimos qué quería decir aquello de El Salvador, país de oportunidades o país de las libertades y ahora no vemos claro el decreto ley de la integración monetaria. Leyendo el articulado de la nueva ley, podemos afirmar que el proyecto de integración monetaria se inicia con un proceso de “desintegración monetaria" a tres niveles diferentes: la desintegración del colón, la moneda nacional, la desintegración de la política monetaria nacional y el peligro de desintegración de la integración económica centroamericana.

Es claro, en primer lugar, que con esta ley, el colón está sentenciado a una pronta eutanasia; poco a poco dejará de ser medida de valor, instrumento de intercambio y sobre todo patrón de pagos diferidos, medio seguro de ahorro. Manuel E. Hinds había escrito, y con bastante razón, que en América Latina tenemos dos monedas: "una es de verdad y otra de mentira. La de verdad es el dólar y la de mentira es la moneda local de cada uno de nuestros países. El dólar es la moneda de verdad porque en ella pensamos en términos de conservación del valor en el tiempo, que es la función principal del dinero. Esto es así porque en América Latina las monedas locales han perdido su valor muchas veces en el pasado, causando graves complicaciones a consumidores, empresarios, trabajadores, ahorrantes e inversionistas" (La Prensa Gráfica, 3 de diciembre de 2000 , p. 6c). El resto del artículo es bien interesante, porque nos dice cosas que son ciertas. También hay que decir que actualmente El Salvador no padecía de elevadas tasas de inflación y el tipo de cambio era fijo. Lo que sí tipificaba a El Salvador es que la mitad de la población no tenía poder de compra, ni siquiera 
en moneda nacional, y eso no se arregla con sólo la dolarización.

Junto con ley de integración monetaria aparece una doble página de preguntas y algunas respuestas curiosas. A la pregunta 13 se responde: "hasta hoy han existido dos monedas en el país: la moneda de los ricos (el dólar) y la moneda de los pobres (el colón)". Esto es cierto, pero no del todo. Desde la década de los ochenta, el mayor ingreso de dólares son remesas de emigrantes; en 1980 hablábamos del "reciclaje de los pobre-dólares", porque los emigrantes enviaban esos dólares, que otros no pobres compraban en la calle El Correo y los colocaban en Estados Unidos. No es muy exacta la respuesta porque los 1500 millones de dólares de las remesas anuales los crean los pobres de fuera y no tanto los ricos de dentro. De todas formas, este es un simple paréntesis y tal vez sólo cuestión de lenguaje, porque también es cierto que los ricos solı los que usan más dólares.

Es normal que la desaparición del colón genere dudas y traumas en la gran población laboral. Sin extendernos a decir que el colón es uno de los símbolos de la identidad nacional y que el huracán de la globalización tiende a derruir todo rastro de esa identidad, baste recordar que el colón ha sido nuestra "medida de valor", el metro decimal de nuestros intercambios. Su desaparición causa dudas, preguntas y traumas. Es claro que con la nueva ley el colón tiende a desaparecer. La gran masa de moneda escriptural se denomina en dólares: todos los ahorros, todos los depósitos, todos los títulos valores del Banco Central de Reserva, los ahoros de las administradoras de los fondos de pensiones y poco a poco los títulos de la bolsa de valores. También los salarios pueden reclamarse en dólares. No significa esto que el colón se suprime por ley, porque el Artículo 5 dice: "El canje de dólares por colones, sea que lo haga el Banco Central de Reserva de El Salvador a los bancos del sistema o bien éstos a los usuarios de los mismos, no generará ningún tipo de comisión o cargo". Si las transacciones monetarias interbancarias y los depósitos de ahorro personales se denominan y se transan en dólares, es claro que toda la economía se dolariza, Hasta el Ministro de Hacienda dejó escapar la palabra dolarización.

Es aquí donde se prevén uno o dos problemas. Cuando la moneda, que es medida de valor, pierde su valor, los ciudadanos perdemos nuestros valores. Aunque esta frase se aplica a la inflación, la podemos aplicar a este proceso de transición bimonetarista. Tiendas y mercados deben fijar sus precios en dólares y colones, y ya se está hablando del "redondeo" de los precios, por supuesto con tendencia al alza del "centaveo" en dólares. La gente sencilla fácilmente puede ser engañada, pese a las anunciadas medidas de control del Ministerio de Economía... Pero queda la otra vertiente: ien qué dirección se van a redondear los salarios de la clase laboral? Esta sería una buena ocasión para redondear hacia arriba, por los menos, los salarios mínimos, porque nuestro mal mayor es la demanda deprimida de la población. Esto de la dolarización tiene algunas ventajas comparativas. Hasta ahora, estilos de vida diferentes utilizaban monedas o medidas de valor diferentes. Ahora, con la dolarización, un salario mínimo de 1,260 colones equivale a 144 dólares, que es lo que cuesta dormir una noche en uno de los hoteles elegantes. ¿La productividad de un mes de trabajo será igual a la productividad de una noche de hotel? Desde luego, nuestra economía practica dos medidas de valor y ¿hará algo más la dolarización?

En segundo lugar, desintegra la política monetaria. El Banco Central de Reserva deja de ser el banco de la nación, banco de bancos y prestamista en última instancia. Es cierto que en el pasado el Banco Central de Reserva ha estado más preocupado por el control de la inflación que por la promoción del empleo. También es cierto que la Superintendencia Financiera no advirtió o corrigió a tiempo claros delitos financieros, que ensuciaron la imagen del sistema y la confianza en las instituciones de captación de ahorro y concesión de créditos. Se pierden prácticamente las principales medidas de política monetaria en un momento en que se venía hablando y pidiendo la autonomía y apoliticidad del Banco Central de Reserva. ¿Cómo es que se cambia tan de repente la misión y objetivos fundamentales de un banco central? Esto sí es un "golpe de timón", públicamente criticado por Juan Héctor Vidal, entre otros académicos.

Lo que está sucediendo con el Banco Central de Reserva me recuerda lo que el Congreso de Estados Unidos quería hacer con el Fondo Monetario Internacional y el Banco Mundial, en la reunión de primavera de estas instituciones (abril del 2000) y en la reunión de Praga (septiembre del 2000), para decir que son cosas de hoy y ahora. Todos sabemos que el Fondo Monetario Internacional y quizás, en menor medida, el Banco Mundial son 
culpables de muchos daños y errores en la aplicación de sus políticas monetarias y financieras, en los países en desarrollo. La teoría del Congreso (comisión Meltzer) era reducir a su mínima expresión el poder de ambas instituciones. Parece que se ha impuesto la tesis de que los errores del pasado no se corrigen con "menos Fondo Monetario Internacional y menos Banco Mundial", sino con "otro Fondo Monetario Internacional y otro Banco Mundial", controlados y sometidos a una superintendencia clara y transparente. Eso es lo que ha ratificado y ojalá sea para bien de los países subdesarrollados, que no son atendidos por el "mercado de los grandes capitales privados" y no son sujetos de su atención. Con nuestro banco central ha triunfado la tesis del Congreso estadounidense y de Larry Summers. Lo que necesitábamos era "otro banco central", pero no un mini-banco central, encargado de informar a la Superintendencia del Sistema Financiero del porcentaje de reservas de liquidez de los bancos y de elaborar las estadísticas económicas del país, que ojalá ahora sí sean más transparentes y fidedignas.

Por estos días, nuestra suprema autoridad monetaria, Alan Greespan, analiza si conviene bajar un poco la tasa de interés de Estados Unidos. En los nueve meses pasados la tasa de interés subió un poco en seis ocasiones para frenar posibles amagos de inflación, controlando así la "burbuja" de la bolsa de valores. Como la economía de Estados Unidos da signos de cierta desaceleración, tal vez se pueda bajar un poco su tasa de interés. Las medidas de "nuestra suprema autoridad monetaria" se reflejarán en la nueva integración monetaria...

Con un golpe de timón nos quedamos sin política cambiaria y sin política monetaria; nos queda la política fiscal. De acuerdo a fragmentos informativos, el Fondo Monetario Internacional dio su "apoyo condicionado a que el plan del Estado venga acompañado de medidas de política fiscal". Literalmente, se cita esta recomendación de Horst Köhler: "si ese plan va acompañado de las medidas de política fiscal que ahora son consideradas por la asamblea y continúan los esfuerzos por mejorar la supervisión bancaria, ayudará a EI Salvador en la promoción del crecimiento económico" (El Diario de Hoy, viernes, 24 de noviembre de 2000 , p. 49). El plan del Estado depende de dudosas condiciones. Primero, que la Asamblea Legislativa "considere", es decir, analice, evalúe y pondere atentamente la estructura del presupuesto es- tatal y no lo resuelva con un "paquetazo" de mano alzada, como parece vislumbrarse. Segundo, que el Estado entienda que el éxito o el fracaso dependen, en buena medida, de su política fiscal. El manifiesto de la Fundación Salvadoreña para el Desarrollo Económico y Social (FUSADES) señala como primera condición del éxito de la dolarización: "una disciplina fiscal para que las finanzas públicas se equilibren y que las autoridades estén en condiciones de implementar una política fiscal que produzca ahorro público en las épocas de bonanza y lo utilice en las difíciles" (La Prensa Gráfica, 30 de noviembre de 2000 , p. 45 ).

Como en los tres últimos años no se ha cumplido con estos requisitos, el presidente Flores se lo recuerda a sus colegas del gobierno: "habrá para nuestro gobierno tres exigencias fundamentales. La primera es la rigurosa supervisión del sistema financiero. La segunda es el cobro efectivo de impuestos, y la tercera es la agilidad en la inversión pública" (La Prensa Gráfica, 6 de diciembre de 2000 , p. 25). Tres exigencias y tres puntos suspensivos para el $2001 \ldots$

En tercer lugar, hay que cuestionar si la integración monetaria no implica la desintegración centroamericana. Al tratado de libre comercio con México vamos tres países juntos; en el proceso de dolarización caminamos por cuenta propia y en forma inconsulta con el resto de socios centroamericanos. Es cierto que, luego de tantas cumbres de presidentes y reuniones de técnicos ministeriales, la integración centroamericana sigue siendo una carrera con vallas. Seguimos siendo estados desunidos de Centroamérica, donde cada cual busca sus alianzas bilaterales. Desde este punto de vista, habría una razón para "ir por su cuenta". De todas formas, estos países son nuestros más próximos socios comerciales, y con ellos - como grupo- mantenemos una balanza comercial deficitaria, sobre todo con Guatemala y Costa Rica. El 23 de noviembre estalló la protesta de nuestros exportadores. Nuestro tipo de cambio anclado y relativamente sobrevaluado restará competitividad a nuestras exportaciones y beneficiará a nuestros socios cercanos con su tipo de cambio flotante o programa de mini-devaluaciones. Queda en suspenso si la anunciada reducción de nuestra tasa de inflación podrá aliviar, en parte, la disparidad cambiaria. Tampoco podemos presagiar qué otras reacciones comerciales o arancelarias puedan adoptar nuestros socios cercanos ante la repentina opción de 
nuestro gobiemo. ¿Se abrirá Guatemala a la dolarización? El único dato real es que tenemos una balanza deficitaria con este bloque de países.

\section{El boomerang de la dolarización}

La ley de la integración monetaria puede regresar, a modo de boomerang, contra el gobierno que la editó y los partidos que la aprobaron. Dejando de lado estos altercados de la Asamblea Legislativa, que ni se olvidan, ni se perdonan, el gobierno ha generado demasiadas expectativas que, de no ser realistas, se traducirán en protestas y manifestaciones cívicas y civiles. Más allá de las expectativas están las dificultades reales de una economía "entrampada y estancada". En este sentido, el manifiesto de la Fundación Salvadoreña para el Desarrollo Económico y Social (FUSADES) es realista. "Pero la dolarización de la economía no será suficiente por sí sola para la reactivación...". El gran desafío es hacer que la dolarización funcione lo más eficazmente posible en el mediano y largo plazo. Para ello, FUSADES recomienda: disciplina fiscal, solidez y supervisión del sistema financiero, flexibilidad de los mercados para que el país pueda amortiguar impactos negativos externos y líneas crediticias internacionales, que le permitan al país enfrentar contingencias financieras y, de esta manera, sustituir - al menos parcialmente- el tradicional papel del Banco Central de Reserva de prestamista de última instancia...
El proyecto de dolarización no debe sobredimensionarse como reactivador de la economía y no debe ser motivo para no continuar impulsando las transformaciones institucionales fundamentales siguientes: "la modernización del Estado, el aumento del acceso y mejora de calidad de los servicios sociales, muy especialmente de educación y salud y la reducción del crimen y mejora de la seguridad ciudadana" (La Prensa Gráfica, 30 de noviembre de 2000 , p. 45) .

El presidente Flores cerró su discurso pidiendo optimismo y prudencia. Es difícil que tengamos optimismo ante un gobierno que no da muestras de mucha prudencia. Ya hemos sido testigos de dos quinquenios de lo mismo: poco optimismo y poca prudencia. Esto no quita que nos esforcemos por cumplir nuestras respectivas tareas, porque aquí se juega la suerte del pueblo, y porque creemos que el pueblo va a exigir el cumplimiento de tantas promesas. Si la economía está entrampada, ello es culpa de quienes han dirigido y han controlado la conducción del modelo para sus propios fines. Nuestro historiador nos contará, Dios mediante, cómo acabó todo en el año 2010. Ojalá diga que el proceso de dolarización comenzó como una "concertación mutilada".

Francisco J. Ibisate S. J. Decano de Ciencias Económicas y Sociales Universidad Centroamericana "José Simeón Cañas" 